\title{
THE PHESAT95 CAMPAIGN OF OBSERVATIONS OF THE PHENOMENA OF THE SATURNIAN SATELLITES
}

\author{
J.-E. ARLOT , W. THUILLOT , F. COLAS , P. DESCAMPS , \\ J. BERTHIER, B. MORANDO , C.H. VEIGA, D.T. VU AND \\ CH. RUATTI \\ Bureau des longitudes \\ Unité de Recherche Associée au CNRS URA 707 \\ 77 avenue Denfert-Rochereau, 75014 PARIS, FRANCE \\ J. LECACHEUX \\ Observatoire de Meudon \\ 5 Place J. Janssen, 92195 MEUDON, FRANCE \\ AND \\ P. LAQUES \\ Observatoire Midi Pyrénées \\ Observatoire du Pic du Midi \\ BAGNËRES DE BIGORRE, FRANCE
}

\begin{abstract}
This paper reports on the international PHESAT95 campaign of observations of the Saturnian events coordinated by Bureau des longitudes. Thanks to CCD or photometric receptors, accurate astrometric data can be get from the observation of the eclipses by Saturn and mutual events of the Saturnian satellites. These events occur from 1994 to 1996 and we give our first results.
\end{abstract}

\section{Introduction}

Every 15 years, the Earth and the Sun cross the equatorial plane of Saturn. Since the rings become less bright, various observations may be performed close to these events: research of new satellites, study of the faint ring structures, astrometry of the faint satellites. Furthermore during about three years around the plane crossing events, eclipses of the satellites by Saturn and mutual occultations and eclipses are observable. In advance to the exploration of the Saturnian system by the CASSINI space probe, these 
events are the occurrence to get accurate astrometric measurements of the Saturnian satellites. They are also a rare opportunity to get information on some physical parameters related to the surface parameters of these satellites.

\section{The PHESAT95 Campaign}

We have organized an international campaign to observe the eclipses and mutual events of the Saturnian satellites. Observers using photometric or CCD receptors have been called to join this campaign. A workshop was organized jointly by Bureau des longitudes and the Bucharest Institute of Astronomy and was held in Bucharest in September 1994. Problems related to acquisition and treatment of data were discussed. The proceedings are under publication but they are already available on the Web Server of Bureau des longitudes (http://www.bdl.fr/PHESAT95/phesat95.html). Predictions of the most observable phenomena have already been published (Arlot and Thuillot, 1993). Aksnes and Dourneau (1995) have also published their own predictions. More recently a full list of phenomena, including grazing events, have been made available on the ftp anonymous server $\mathrm{ftp}: / / \mathrm{ftp} . \mathrm{bdl} . \mathrm{fr} / \mathrm{pub} / \mathrm{ephem} / \mathrm{satel} / \mathrm{phesat} 95$, or on floppy disk on request to the authors. A Web page at the address http://www.bdl.fr/phemu gives access to similar informations using the HTML facilities.

\section{First observations}

According to the predictions (Arlot and Thuillot, 1993),(Thuillot and Arlot, 1995), the main period of observations of eclipses and mutual events of the Saturnian satellites begins in summer 1995 and ends early in 1996. But some eclipses by Saturn were observed in 1994. These events are generaly difficult to observe because of the close distance to Saturn. Nevertheless several stations allow this type of observation. This is the case of Pic du Midi Observatory were we success to observe several such events. For example, figure 1 shows the reappearance of Rhea from eclipse by Saturn on November 1994. This event occurred at about 5 arcsec. from the edge of the planet and was observed with a CCD camera (THX7863) on the 1 meter telescope of Pic du Midi Observatory.

More recently mutual events have been observed in several sites. In France, at Haute-Provence Observatory, on June 17 1995, we success to observe the eclipse of Tethys by Enceladus at the 0.80 meter telescope (fig.2), using a CCD camera (THX7863). Such events do not involve the atmosphere of Saturn, contrarily to the eclipses of the satellites by the planet, so we expect to get a high accuracy in the astrometric measurement inferred from this photometric observation. According to previous similar 


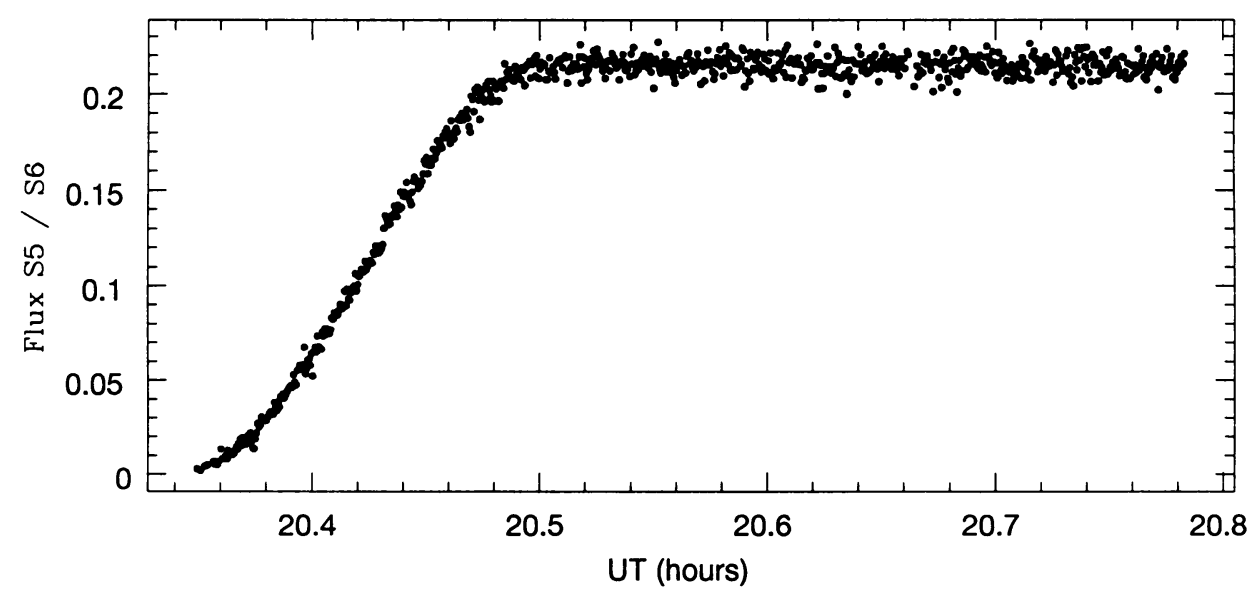

Figure 1. Reappearance of Rhea after an eclipse by Saturn observed on November 1994 at the $1 \mathrm{~m}$ telescope of Pic du Midi Observatory

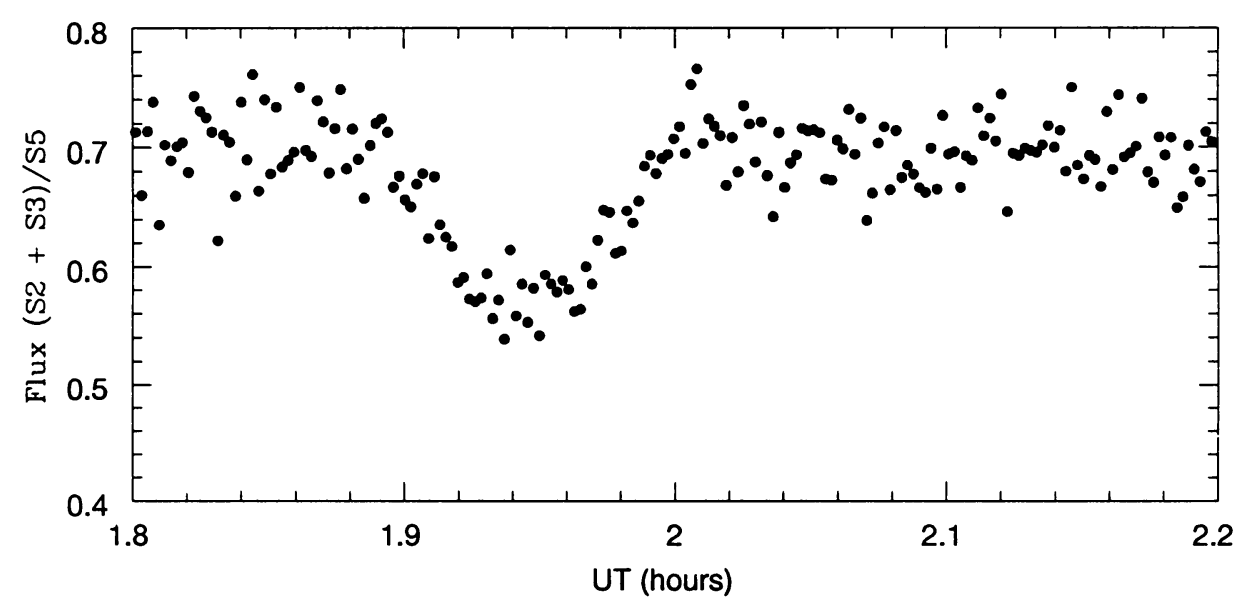

Figure 2. Occultation of Tethys by Enceladus observed on June 171995 at the $0.8 \mathrm{~m}$ telescope of Haute-Provence Observatory 
observations we made concerning the Galilean system of Jupiter, a precision better than $200 \mathrm{~km}(0.03$ arcsec.) can be expected. Furthermore some surface parameters of the satellites could be deduced from the modelling of these events.

\section{Conclusion}

The period of phenomena of the Saturnian satellites, which mainly occur from 1994 to 1996, is a great opportunity to get accurate astrometric measurements of these satellites. The PHESAT95 campaign of observation that we organize will get such data. Subsequently, they could be used in adjustment of the dynamical model of the motion of the Saturnian satellites.

\section{References}

Aksnes, K., Dourneau, G. (1994) Icarus, Vol. no. 112, p. 545-548

Arlot, J.-E., Thuillot, W. (1993) Icarus, Vol. no. 105, p. 427-440

Thuillot, W., Arlot, J.-E. (1995) Proceedings of the Workshop CCD et récepteurs photométriques appliqués à l'observation des satellites de Saturne durant la période favorable de 1994-1996, held in Bucharest, Bureau des longitudes and Institute of Astronomy, (also available on the Web Server http://www.bdl.fr/PHESAT95/phesat95.html) 